\title{
An international registry of patients with plasminogen deficiency (HISTORY)
}

Haematologica 2020

Volume 105(3):554-561

\section{Correspondence:}

AMY D. SHAPIRO

ashapiro@IHTC.org

Received: October 22, 2019.

Accepted: January 20, 2020.

Pre-published: January 30, 2020.

doi:10.3324/haematol.2019.241158

Check the online version for the most updated information on this article, online supplements, and information on authorship \& disclosures: www.haematologica.org/content/105/3/554

(C)2020 Ferrata Storti Foundation

Material published in Haematologica is covered by copyright. All rights are reserved to the Ferrata Storti Foundation. Use of published material is allowed under the following terms and conditions:

https://creativecommons.org/licenses/by-nc/4.0/legalcode. Copies of published material are allowed for personal or internal use. Sharing published material for non-commercial purposes is subject to the following conditions:

https://creativecommons.oró/licenses/by-nc/4.0/legalcode, sect. 3. Reproducing and sharing published material for commercial purposes is not allowed without permission in writing from the publisher.

\section{Amy D. Shapiro, ${ }^{1}$ Marzia Menegatti, ${ }^{2}$ Roberta Palla, ${ }^{3}$ Marco Boscarino, ${ }^{2}$ Christopher Roberson, ${ }^{1}$ Paolo Lanzi, ${ }^{4}$ Joel Bowen, ${ }^{5}$ Charles Nakar, ${ }^{1}$ Isaac A. Janson ${ }^{1}$ and Flora Peyvandi ${ }^{2,3}$}

${ }^{1}$ Indiana Hemophilia \& Thrombosis Center, Indianapolis, IN, USA; 'Fondazione IRCCS Ca' Granda Ospedale Maggiore Policlinico, Angelo Bianchi Bonomi Hemophilia and Thrombosis Center and Fondazione Luigi Villa, Milan, Italy; ${ }^{3}$ Università degli Studi di Milano, Department of Pathophysiology and Transplantation, Milan, Italy; ${ }^{4}$ Misto s.r.l., Milan, Italy and ${ }^{5}$ Rho, Inc., Durham, NC, USA

\section{ABSTRACT}

P lasminogen deficiency is an ultra-rare multisystem disorder characterized by the development of fibrin-rich pseudomembranes on mucous membranes. Ligneous conjunctivitis, which can result in vision impairment or loss, is the most frequent symptom reported. Affected systems may also include the respiratory tract, oropharynx, female reproductive tract, gingiva, middle ear, renal collecting system, skin and central nervous system. Untreated, plasminogen deficiency may result in significant reduction in quality of life and morbidity with potential life-threatening complications. Non-specific therapies are inadequate and plasminogen concentrates are not commercially available. The current understanding of plasminogen deficiency and management of disease symptoms and its progression are based on case reports/series and two small clinical trials. To date there has never been a comprehensive, international study to examine the natural history or optimal therapeutic intervention; knowledge gaps include identification of contributing factors and triggers of disease manifestations, inability to predict disease course, and insufficient real-world data for use of therapeutics. We have created an international, observational study (HISTORY) in a large cohort of persons with plasminogen deficiency and first-degree family members to address these gaps and to advance knowledge and care. HISTORY will build upon the established relationship between the Indiana Hemophilia and Thrombosis Center and the Fondazione Angelo Bianchi Bonomi, IRCCS Ca' Granda Ospedale Maggiore Policlinico - University of Milan and will utilize a modified version of the Prospective Rare Bleeding Disorders Database (PRO-RBDD). A biorepository containing samples from subjects with plasminogen deficiency will be established. This article describes the rationale behind the study and efforts towards its goals.

\section{Introduction}

Diseases are defined as rare if they affect fewer than 200,000 people in the United States of America (USA) or fewer than 1 in 2,000 in the European Union; there are estimated to be approximately 6,500 clinically distinct rare diseases across all medical specialties. ${ }^{1}$ Given the large number of diseases meeting this broad classification, the term 'ultra-rare disease' has emerged to describe the rarest of these disorders; this phrase is not well defined, but in the United Kingdom (UK) it has come to mean fewer than 1,000 patients in that country (approximately 1 in $65,000)$. These diseases pose unique considerations for the researcher, clinician and patient; these range from an understanding of the natural history of the disease and accurate diagnosis, to safe and effective treatment options and availability of knowledgeable specialists.

Randomized and large prospective clinical trials are often not feasible for ultrarare diseases as the limited number of eligible patients and phenotypic hetero- 
geneity affect recruitment, interpretation of data and applicability of conclusions to the larger real-world population of patients; in many cases it may not be possible to design a clinical trial accurately because of insufficient knowledge of the disease. ${ }^{2}$ Instead clinicians have often relied on personal experience, case studies/series and patient registries to understand the natural history of these diseases and to collect data on therapeutic interventions and patient outcomes. Patient registries may be national, regional or international, each with its own advantages and disadvantages. The value of international patient registries in rare diseases has long been recognized; however, given the large number of rare diseases and the small number of affected patients, funding opportunities are frequently limited, particularly for long-term maintenance. ${ }^{1}$

Some diseases are so rare that many clinicians may never see a case; for example, plasminogen deficiency (PLGD) is an ultra-rare disease with an estimated prevalence of approximately 1.6 per million population. Most commonly it presents as ligneous conjunctivitis, an extravascular accumulation of fibrin-rich, woody (ligneous) pseudomembranes on the mucous membrane of the eye; it can also manifest on other mucous membranes, and as a life-long, systemic disease impacting multiple systems either intermittently or continuously. No specific therapies are approved by the Food and Drug Administration or European Medicines Agency and nonspecific treatments are inadequate.

Current knowledge of PLGD stems from case reports and small series; systematic prospective data collection coupled with serial biological samples collected from persons with PLGD and first-degree family members has not been performed. Knowledge gaps include identification of contributing factors to and triggers of disease manifestations, inability to predict disease course, and insufficient real-world data for individualized use of potential new therapeutics. To address unmet needs, a retrospective and prospective data collection system of a large cohort of people with PLGD and their family members has been developed to define the natural history of PLGD and is entitled Hypoplasminogenemia: An International RetroSpecTive and PrOspective CohoRt StudY (HISTORY). HISTORY addresses key gaps in the knowledge about the natural history of PLGD and builds on the productivity/infrastructure of the established collaborative efforts of research teams at the Indiana Hemophilia and Thrombosis Center (IHTC), the Fondazione Angelo Bianchi Bonomi, IRCCS Ca' Granda Ospedale Maggiore Policlinico of Milan (IRCCS) and the University of Milan (UNIMI). Phenotypic data combined with genetic and advanced laboratory testing will be used to investigate disease course predictors and evaluate/elucidate phenotypic relationships. A specimen biorepository will serve as a resource for further analyses. Overarching study goals are to analyze phenotypic heterogeneity, to identify markers to predict disease course, and to develop improved methods to utilize new therapeutics.

\section{Plasminogen deficiency}

Congenital PLGD is an autosomal recessive disorder caused by mutations in PLG that result in functionally deficient and/or reduced levels of circulating plasminogen. ${ }^{3}$ An epidemiological study performed in the UK observed a $0.26 \%$ prevalence of asymptomatic heterozygous PLGD; ${ }^{4-6}$ similar prevalences of heterozygous PLGD have been reported in other countries. ${ }^{7-9}$ The UK data suggest that the prevalence of individuals with symptomatic PLGD (homozygous/compound heterozygous) is 1.6 per million population; ;0,11 there are estimated to be 500 symptomatic people with PLGD in the USA and 12,000 worldwide. Based on experience with other rare bleeding disorders, a higher prevalence of PLGD is likely to occur in regions where consanguineous marriages are more common; ${ }^{11,12}$ for this reason, a national study would likely misstate the true prevalence and phenotypic diversity of the disease. An increased number of females are reported to be symptomatic compared to males (ratio of 1.27-1.88:1)..$^{12,13}$

People with PLGD may exhibit a multi-organ systemic disease that commonly manifests as the extravascular accumulation of fibrin-rich, woody (ligneous) pseudomembranes on mucous membranes. PLGD is a lifelong disease, although the most severe symptoms are observed in infants and children. ${ }^{3,14}$ Ligneous conjunctivitis is the most common disease symptom ( $81 \%$ prevalence); ${ }^{3}$ pseudomembrane growth may be triggered by a local infection or injury and can result in impairment or loss of vision if untreated and/or persistent. ${ }^{3,11}$ Approximately one-third of people with PLGD have corneal involvement with potential for blindness, ${ }^{11,12}$ and $30 \%$ present with ligneous gingivitis resulting in periodontal destruction and tooth loss. ${ }^{3,12,13}$ Other common symptom locations include the respiratory tract (20\%), ears $(14 \%)$, female genitourinary tract $(9 \%)$, and kidneys (4\%). ${ }^{3,11,15-18}$

Lesions are often inflamed and painful and can compromise organ function, resulting in life-threatening conditions including renal and respiratory failure (e.g., tracheobronchial lesions). ${ }^{11,18}$ Central nervous system complications have been noted, including Dandy-Walker malformation and occlusive hydrocephalus ${ }^{12}$ in $14 \%$ of children due to fibrin deposition in the cerebral ventricular system; shunts used to drain cerebrospinal fluid are prone to occlusion or poor peritoneal absorption, leading some neurosurgeons to recommend ventriculocholecystic shunts. ${ }^{19}$ In women, dysmenorrhea, abnormal menses, dyspareunia, and infertility have been reported. ${ }^{15}$ Wound healing may be severely impaired.$^{20}$ Lesions in the middle ear may result in hearing loss. ${ }^{11}$ Although the initial case of PLGD was documented in a patient with recurrent venous thromboembolism, an association between PLGD and increased thrombotic risk has not been demonstrated. ${ }^{10,21}$ Patients may suffer substantial morbidity and mortality and experience reduced quality of life and education/work potential. ${ }^{14}$

Surgical removal of lesions often results in accelerated pseudomembrane regrowth compounding clinical morbidity. ${ }^{22}$ Case reports describing use of topical or systemic heparin, corticosteroids, cyclosporine, azathioprine, hyaluronidase, $\alpha$-chymotrypsin, oral contraceptives, warfarin, and amniotic membrane placement have been published; these approaches show inconsistent success (examples of therapies utilized are shown Table 1). . $^{13,23-29}$ Fresh-frozen plasma has been reported to be successful in some cases when eye drops, subconjunctival injection and/or intravenous infusions are utilized; $;^{30,31}$ however, as fresh-frozen plasma contains low concentrations of plasminogen, volume overload may occur when the intra- 
venous route of administration is used. Infusion reactions, time required for administration and need for repeated infusions may also limit intravenous use of fresh-frozen plasma.

Several case studies have reported on topical ophthalmological application of plasminogen for the treatment of ligneous conjunctivitis. Watts et al. noted that following surgical removal of pseudomembranes, plasminogen eye drops administered every $2 \mathrm{~h}$ prevented regrowth; a maintenance dose every $6 \mathrm{~h}$ prevented symptoms for over 1 year. $^{32}$ Similarly, Pons et al. reported resolution of ligneous conjunctivitis with administration of plasminogen eye drops; in this patient, multisystem manifestations subsequently developed that could not be treated. ${ }^{33}$ An interim analysis of a phase II/III clinical trial studying ophthalmological plasminogen (2 drops, 4-12 times daily) was reported by Nakar et al. ${ }^{34}$ Ten out of 11 subjects (91\%) experienced full regression of ophthalmological lesions and/or absence of recurrence following surgical excision (the $11^{\text {th }}$ subject was withdrawn because of noncompliance). This clinical trial (NCT01554956) is ongoing.

Shapiro et al. have reported results from a phase II/III clinical trial (NCT02690714) investigating the use of an intravenous plasma-derived plasminogen concentrate (6.6 $\mathrm{mg} / \mathrm{kg}$ iv, every 2-4 days depending on individual pharmacokinetic profile). ${ }^{14}$ All 14 subjects achieved trough plasminogen levels at least $10 \%$ above baseline; all preexisting clinical manifestations of PLGD (including lesions on the conjunctiva, gingiva, nasopharynx, bronchus, colon, kidney, cervix, and vagina) resolved or improved. An amended biologics license application is expected to be filed with the Food and Drug Administration for this product in the first half of 2020 .

The rarity of PLGD results in frequent mis- and delayed diagnoses by professionals lacking specialist knowledge. Difficulty with diagnosis and disease rarity have contributed to an inability to document the natural history, develop clinical guidelines, and optimize treatment regimens. An exclusively USA-based registry would be of limited value as this would restrict recruitment and might fail to identify the full spectrum of the disorder. Therefore, an international effort has been undertaken to gain unique and broad insights into the disease, its progression and optimal treatment.

\section{Registries in rare coagulation disorders}

Hemophilia A and B are two of the more common rare coagulation disorders; they affect approximately 1:5,000 and 1:30,000 persons, respectively. Our current understanding of hemophilia and the wide availability of specialized care and therapeutic options are a result of the many clinical trials and patient registries that have been developed over the years.

Patient registries in hemophilia may be national, regional or international. O'Mahony et al. noted that 27/35 European countries had their own national hemophilia patient registry and some countries have multiple national registries operated by different organizations with little consideration given to interoperability. ${ }^{35,36}$ This lack of interoperability and the variability in inclusion/exclusion criteria for enrolled subjects, definitions utilized, data collected, and different goals of these
Table 1. Examples of published therapies for the most common manifestations of plasminogen deficiency, ligneous conjunctivitis and ligneous gingivitis.

\begin{tabular}{|c|c|c|}
\hline Therapy* & $\begin{array}{c}\text { LC } \\
\text { [reference] }\end{array}$ & $\begin{array}{c}\text { LG } \\
\text { [reference] }\end{array}$ \\
\hline Fresh-frozen plasma & [31] & {$[22]$} \\
\hline Plasminogen concentrate & {$[14,34]$} & [14] \\
\hline $\begin{array}{l}\text { Anticoagulants } \\
\text { Heparin } \\
\text { Warfarin } \\
\text { Argatroban }\end{array}$ & $\begin{array}{l}{[23]} \\
{[33]}\end{array}$ & [28] \\
\hline $\begin{array}{l}\text { Immunosuppressants } \\
\text { Corticosteroids } \\
\text { Azathioprine } \\
\text { Cyclosporine A }\end{array}$ & $\begin{array}{c}{[23]} \\
{[11]} \\
{[24,25]}\end{array}$ & [10] \\
\hline Oral contraceptives & {$[27]$} & \\
\hline $\begin{array}{l}\text { Thrombolytic } \\
\text { Anistreplase }\end{array}$ & [10] & \\
\hline Hyaluronidase & {$[26]$} & \\
\hline Alpha chymotrypsin & {$[26]$} & \\
\hline $\begin{array}{l}\text { Mast cell stabilizer } \\
\text { Cromoglicic acid }\end{array}$ & {$[11]$} & \\
\hline $\begin{array}{l}\text { Anti-proliferative agent } \\
\text { Mitomycin C }\end{array}$ & [11] & \\
\hline
\end{tabular}

LC: ligneous conjunctivitis; LG: ligneous gingivitis. *Not all reported therapeutic results have been shown to be reproducible.

national registries all limit the pooling of data to the detriment of all stakeholders. One example of the issues associated with multiple registries pursuing similar goals may be observed in those that examined the determinants of hemophilic inhibitor development and predictors of immune tolerance induction success: different registries reported different conclusions based on the patients enrolled and the data collected. ${ }^{37}$ Ideally, collaboration between registries and harmonization of data collection are necessary to achieve minimal datasets for advancement of shared clinical goals in these rare disorders. ${ }^{38,39}$

Advantages and disadvantages of national, regional and international registries are highlighted in the following examples.

\section{The American Thrombosis and Hemostasis Network}

The American Thrombosis and Hemostasis Network (ATHN) collects data on patients in the USA with rare coagulation disorders. The centralized database and standardized definitions for data entry enhance research and clinical goals; these include the generation of the largest genetic hemophilia repository (My Life, Our Future) and the surveillance of over 80,000 persons with rare coagulation disorders (Community Counts). ${ }^{40,41}$ Despite these successes, Gupta et al. noted limitations in data collection; for example, of the known 3,626 patients with an ultrarare coagulation disorder, less than $11 \%$ had been included in the Community Counts registry. ${ }^{39}$ Furthermore, the strictly USA-centric dataset may limit wider applicability of results to other countries with different medical resources and population diversity. These limitations highlight why efforts to create a comprehensive ultra-rare coagulation disorder registry in a single country may be less successful than desired. 


\section{European Network of Rare Bleeding Disorders}

The Rare Bleeding Disorders Database (RBDD) project was established at UNIMI in 2004 to organize and analyze clinical, genetic and treatment data on rare bleeding disorders, including deficiencies of fibrinogen and factors II, V, combined V and VIII, VII, X, XI and XIII. In 2007, this database was modified to include the use of a webbased application to create the retrospective European Network of Rare Bleeding Disorders (EN-RBD) project. ${ }^{42}$ Data on 592 patients from 11 European countries were included. These data led to the first authoritative understanding of the relationship between coagulation factor activity level and clinical bleeding severity in ultra-rare bleeding disorders; in turn this resulted in the development of a new severity classification system for bleeding symptoms. ${ }^{43}$

\section{Prospective Rare Bleeding Disorders Database}

To overcome limitations of retrospective data collection, EN-RBD was subsequently modified to incorporate prospective data on patients. This registry, the Prospective Rare Bleeding Disorders Database (PRORBDD), enrolled patients from 62 centers in Europe, Asia, the Middle East, the Americas, Africa, and Oceania. ${ }^{44}$ The greatly expanded international network of participating centers was considered essential for a registry focusing on diseases for which the prevalence may approach 1 per million population; this extended outreach may permit increased and more rapid enrollment, and greater phenotypic and genetic diversity.

The aims of PRO-RBDD are a more accurate determination of the prevalence of rare bleeding disorders, the incidence of bleeding episodes, the use of treatment products, and the optimization of clinical management. Currently, subjects with deficiencies in fibrinogen and factors V, V/VIII, XI and XIII are being enrolled in PRORBDD with phenotype/genotype evaluation performed at a central laboratory to ensure consistency. Initial results have helped define minimal factor XIII plasma levels (15 $\mathrm{IU} / \mathrm{dL}$ ) necessary to prevent spontaneous major bleeding in people with factor XIII deficiency; ${ }^{45}$ this observation highlights the importance of registry data to clinical practice.

\section{The plasminogen deficiency registry (HISTORY project)}

HISTORY is an extension of PRO-RBBD; it is an observational cross-sectional study that will contain both retrospective and prospective data from an international population of people with PLGD and their immediate family members. PRO-RBDD was selected as the ideal infrastructure for HISTORY as previously gained experience, proven track-record and established collaborations should ensure timely and efficient achievement of study goals. Retrospective data will be collected for 1 year prior to study entry; prospective data will be collected for each subject for a 3-year on-study period.

The overarching goals driving data analysis are development of disease severity categories, disease course predictors and need for specific surveillance in particular subpopulations, and treatment algorithms and recommendations to guide clinical care and management. As such, the registry design will allow collection of general information about each subject's health, with specific details regarding: original diagnosis (age, reason for screening); phenotype and genotype analysis; type, site and number of clinical manifestations indicative of pseudomembrane formation; detailed information on type, intensity, and duration of any prophylactic treatment, frequency and dose; type, frequency and dose of therapy administered to treat acute or chronic pseudomembranes; laboratory parameters; use of concomitant therapy; detailed information of management of surgical procedures; obstetric data; and complications associated with treatment.

The coordinating centers for this study are the IHTC and IRCCS/UNIMI. The IHTC is enrolling subjects from North, South and Central America and IRCCS/UNIMI is enrolling subjects from the rest of the world. The database will be located at UNIMI and specimens will be stored at the IHTC and/or IRCCS/UNIMI biorepositories. Safeguards have been implemented to protect confidential medical information to meet national regulations; all clinical information and biological samples will be stored in accordance with the Health Insurance Portability and Accountability Act of 1996 or General Data Protection Regulation in the USA and Europe, respectively.

Clinical study protocols and laboratory manuals have been developed and approved by local institutional review boards. HISTORY will be conducted in compliance with Good Clinical Practice as stated in the Declaration of Helsinki; it is registered at clinicaltrials.gov (NCT03797495).

\section{Patient recruitment and study visits}

There is currently no authoritative natural history study for PLGD; HISTORY is a 4-year study (Figure 1A) that will be the first international effort to define the natural history of this disorder by reviewing retrospective and prospective data from up to 100 probands and their firstdegree family members (approximately 500 subjects in total). Asymptomatic family members will be recruited in addition to symptomatic individuals. Asymptomatic family members are not routinely tested for PLGD; study testing will provide a unique opportunity to prospectively monitor any newly diagnosed patients to investigate the deficiency prior to symptom development. The inclusion of heterozygous family members may additionally reveal the relationship between minimal plasminogen activity levels and natural history. Inclusion and exclusion criteria are listed in Table 2.

The registry will include a minimum of seven data entry points per subject (baseline and every 6 months for 3 years) with data also collected at other non-scheduled visits (Figure 1B and Table 3). Retrospective baseline data (demographic and clinical history for 1 year prior to enrollment) will be collected at each study site, with prospective follow-up data acquired by telephone if an in-person visit is not required. In-person evaluation will occur in cases of suspected clinical manifestations indicative of pseudomembranes or other intermittent medical events including pregnancy. Laboratory evaluation and physical examination will be performed at baseline and at study termination.

To accelerate recruitment and meet the study objectives efficiently, PLGD patients/families/providers held within an IHTC tracking system ( $\sim 50$ probands) will be contacted to determine interest in the study, as will authors of published literature on cases/series of PLGD. Advertisements will be placed in scientific journals that focus on diseases for which clinicians may interface with people with PLGD. Furthermore, an outreach program using social media, a 
dedicated website ( $w$ ww.plgdeficiency.com), presentations at international meetings, and direct contact with interested colleagues will be pursued. Study coordinators at IRCCS/UNIMI will contact their established treatment center network throughout the world. In addition to hematologists, specialists including gynecologists and ophthalmologists will also be contacted; these healthcare professionals may be the first to observe a patient with PLGD. A multidisciplinary approach including such specialists is essential to improve overall awareness and understanding of the disorder; it may also prevent the loss of patients to follow-up by a hematologist as some patients may continue treatment outside of a specialist hematology center. As PLGD may affect different systems throughout life, the investigators are enrolling subjects across a wide range of ages, countries, phenotypes and genotypes. People with PLGD are motivated to participate in studies to advance PLGD understanding as few study opportunities exist.
To date, 26 centers from 12 countries have agreed to participate in HISTORY; 51 subjects (24 probands and 27 first-degree family members) are currently enrolled in the study. Demographic data for these 51 subjects are shown in Table 4. Initial results have identified asymptomatic siblings with PLGD; these subjects are being monitored to understand the factors that trigger initial symptom development.

\section{Therapeutic interventions}

Regular monitoring and individualization of therapy is required for people with PLGD as there is currently no compelling evidence that a patient's clinical course can be predicted from their plasminogen activity level or genetic defect. ${ }^{12}$ Similarly, if a patient presents with ligneous conjunctivitis, the likelihood of developing multisystem disease cannot be predicted; it is unknown what routine scans should be performed, what surveillance methods

\section{A Overall study timeline}

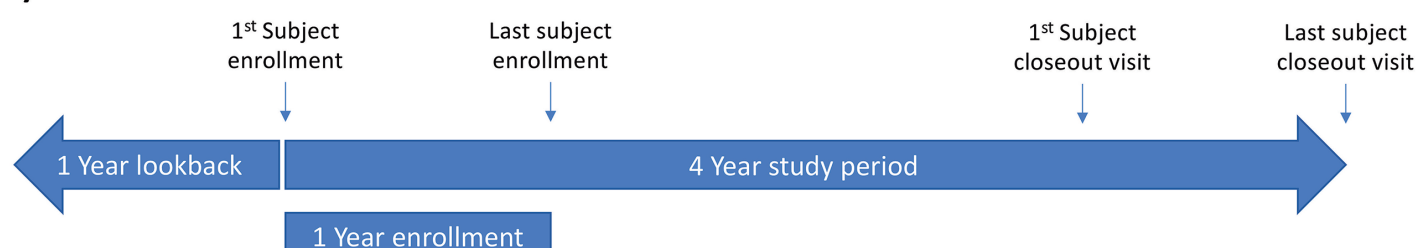

1 Year enrollment

First subject: 3 year on-study period

Last subject: 3 year on-study period

B Subject visits \& data collection

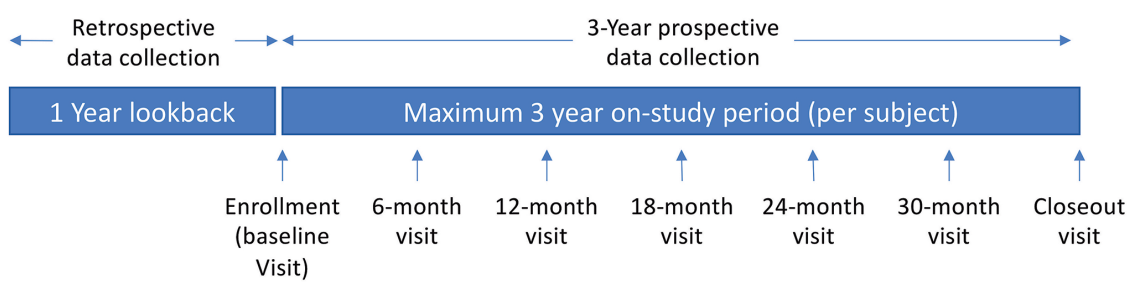

Figure 1. Overall study timeline and scheduled study visits. (A) The 4-year study plan includes a 1-year enrollment period and a maximum 3-year on-study period for each subject enrolled. Retrospective clinical data for 1 year prior to study enrollment will also be collected. (B) The initial baseline visit will include informed consent, demographics, screening, laboratory investigations, genetic testing, medical examination and history, and a 1-year retrospective collection of relevant clinical data. Subsequent study visits will occur every 6 months and will include a medical history review; the closeout visit will occur at the 3 -year time point. See also Table 3.

Table 2. Inclusion and exclusion criteria for enrollment in HISTORY

\section{Inclusion criteria}

Males or females with plasminogen deficiency diagnosed locally with plasminogen activity levels $<50 \%$ OR first-degree family members of a person diagnosed with plasminogen deficiency (to include parents, siblings, half-siblings)

\section{All ages eligible}

Available clinical history and treatment for at least 1 year prior to entry except for infants $<1$ year of age

Willingness to provide samples for analysis including DNA, plasma etc.

Willingness to participate in prospective follow-up for up to 3 years

\section{Exclusion criteria}

Any psychiatric disorder, other mental disorder, or any other medical disorder that impairs the subject's ability to give informed consent or to comply with the requirements of the study protocol (unless a caregiver or authorized representative is willing to provide consent/assent)

Previous organ transplant recipient

Refusal to provide informed consent

Special patient populations, including prisoners or those who are deemed medically or cognitively unsuitable for research by their treating physician Inability to obtain a blood sample due to poor or limited venous access 
Table 3. Details of the study visits.

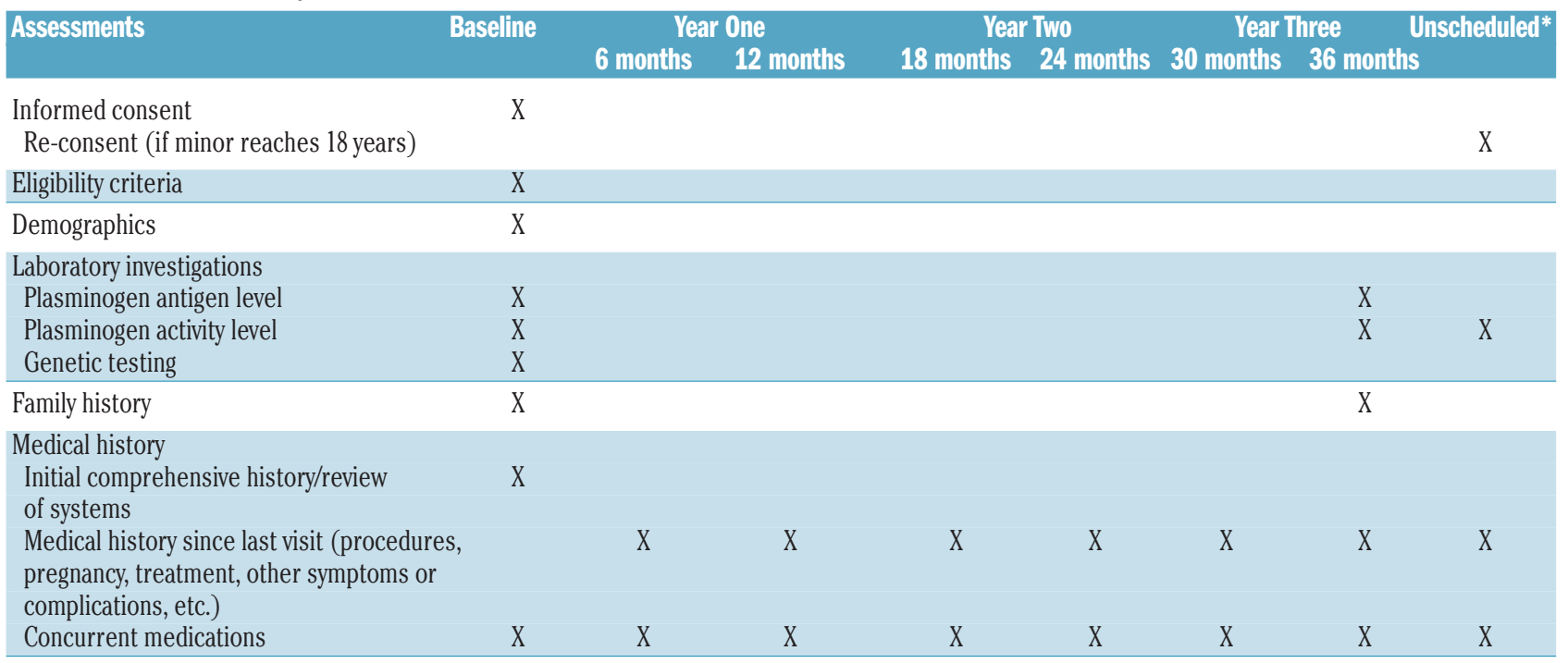

*Additional data will be collected at the time of an unscheduled visit for re-consent, illness, pregnancy (plasminogen activity level and banked plasma will be collected at confirmation of pregnancy, during the third trimester and at delivery), and development of lesions.

should be used, or how frequently clinical visits should occur to monitor for symptoms to ensure early intervention and prevent sequelae. Furthermore, it is unknown if treatment should be lifelong, intermittent, or may be withdrawn over time and how this varies by patient, disease severity, and affected system.

All non-specific therapeutic interventions to treat acute and chronic manifestations of PLGD will be recorded, along with efficacy, safety and complications. Specific treatments, including ophthalmological plasminogen drops and systemic intravenous plasminogen replacement therapy are currently under clinical investigation; $;{ }^{14,34}$ any use of these investigational agents will also be recorded. Currently, data to support wider real-world optimal therapeutic regimens are lacking. There are no standard dosing regimens for intravenous plasminogen replacement therapy and the required minimal plasminogen plasma levels to treat or prevent specific manifestations are unknown; treatment for each patient must be individualized based on their pharmacokinetic profile. ${ }^{14}$ HISTORY will explore development of a population pharmacokinetic model $^{46}$ for the investigational intravenous plasminogen concentrate ${ }^{14}$ to estimate plasma levels and adjust doses if symptoms emerge.

\section{Factors that correlate with disease expression and severity}

No screening test for PLGD exists; specific plasminogen activity/antigen levels are required, but assays are not uniformly available. This study will collect methodological details of locally performed tests to review their diagnostic utility; testing will be repeated centrally to confirm diagnosis and local assay utility. Peyvandi et al. demonstrated that clotting level activity in some rare bleeding disorders does not correlate with clinical bleeding severity. ${ }^{43}$ Published data suggest that this may also be true for PLGD; therefore, global coagulation assays (including thrombin generation, thromboelastography, simultaneous thrombin/plasmin generation and fibrinolytic potential) $)^{47.50}$ will be performed to evaluate their utility in phenotypic prediction and clinical management.
Table 4. Demographics of the initial 51 enrolled subjects.

\begin{tabular}{|c|c|c|}
\hline Demographics & $\begin{array}{c}\text { Subjects with } \\
\text { plasminogen deficiency }\end{array}$ & $\begin{array}{l}\text { Unaffected family } \\
\text { members }\end{array}$ \\
\hline $\begin{array}{l}\text { Gender } \\
\text { Male } \\
\text { Female }\end{array}$ & $\begin{array}{c}9 \\
15\end{array}$ & $\begin{array}{l}11 \\
16\end{array}$ \\
\hline $\begin{array}{l}\text { Age } \\
\quad<18 \text { years } \\
\geq 18 \text { years }\end{array}$ & $\begin{array}{l}14 \\
10\end{array}$ & $\begin{array}{c}8 \\
19\end{array}$ \\
\hline $\begin{array}{l}\text { Ethnicity } \\
\text { White } \\
\text { Hispanic/Latino }\end{array}$ & $\begin{array}{c}21 \\
3\end{array}$ & $\begin{array}{c}22 \\
5\end{array}$ \\
\hline $\begin{array}{l}\text { Consanguineous pa } \\
\text { Yes } \\
\text { No } \\
\text { Unknown }\end{array}$ & $\begin{array}{c}0 \\
22 \\
2 \\
\end{array}$ & $\begin{array}{c}0 \\
27 \\
0\end{array}$ \\
\hline $\begin{array}{l}\text { Endogenous plasmi } \\
\text { Median (\%) } \\
\text { Range (\%) }\end{array}$ & $\begin{array}{c}18.5 \\
1-42\end{array}$ & $\begin{array}{l}n / a \\
n / a\end{array}$ \\
\hline $\begin{array}{l}\text { Endogenous plasm } \\
\text { Median (mg/dL) } \\
\text { Range (mg/dL) }\end{array}$ & tigen & $\begin{array}{l}n / a \\
n / a\end{array}$ \\
\hline
\end{tabular}

The true prevalence of PLGD is not well established and likely depends on the population in any particular region or country; similarly, the genetic alterations influencing disease expression and severity are not well defined. A genome-wide association study conducted by Ma et al. determined genetic modifiers of plasminogen levels in normal adults; ${ }^{51}$ the study revealed that four single nucleotide polymorphisms were associated with variations in plasminogen levels. How these variations affect the severity or progression of PLGD is unknown. The PLG(K38E) mutation is known to result in a milder clinical course; $;^{12,13}$ conversely, other mutations are known to not be predictive (for example, in one family with the conserved W597C mutation, one member was asymptomatic, another had ligneous periodontitis, and a third had 
ligneous conjunctivitis). ${ }^{12}$ HISTORY will record the prevalence of different mutations in the affected and heterozygote population and attempt to determine the relationship between mutation and clinical penetrance heterogeneity; it will also examine polymorphisms that may influence fibrinolytic capacity (e.g., in fibrinogen A $\alpha$ and plasminogen activator inhibitor 1)..$^{52}$ The enrollment of relatives of people with PLGD may enable a refinement of plasminogen activity levels observed in heterozygotes.

The registry will also incorporate environmental and host-specific factors that may modify plasminogen levels and/or disease symptoms. A study in healthy subjects found that smoking contributes to plasma plasminogen levels $;{ }^{51}$ it is not known whether this and other environmental factors affect plasminogen plasma levels in patients with PLGD. It is also not clear whether an environmental trigger is required for symptom development and/or disease expression, or whether and, if so, how specific plasminogen antibodies develop. HISTORY will address some of these unknown environmental/host factors.

\section{Plasminogen deficiency biorepository}

Research involving human genetic or genomic information analyzed in conjunction with personal or health data has become increasingly important for untangling genetic, lifestyle and environmental disease determinants. A biorepository containing DNA, plasma, and serum will be created; samples will be stored for up to 15 years. If planned analyses are not revealing, stored samples will be utilized to explore other biomarkers to elucidate a correlation/prediction of phenotypic heterogeneity (e.g., polymorphisms in inflammatory markers, whole genome analyses).

\section{Data analyses}

To understand the impact of specific data on disease expression, all collected data will be analyzed. Clinical data on people with PLGD will be evaluated for categorical characterization (i.e., asymptomatic, intermittently symptomatic, and continuously symptomatic). The probands' age in each category will be analyzed to evaluate age as a variable for symptom expression. Categories will be modeled and analyzed against a range of variables including gender, environmental factors, genetic studies, laboratory coagulation parameters including specific and exploratory global assays, and treatment administration. Subjects with clinical symptoms will be evaluated for the presence of individual affected sites versus multiple/systemic symptoms, and also analyzed based on prior listed variables. A descriptive analysis of symptoms reported by physiological system will be performed and modeled against study variables (levels, genetics, global assays, and exploratory investigations) to determine whether any of them are predictive. Symptoms and data will be reviewed to determine whether severity categories can be established. Urine analysis will be performed, as unpublished data suggest that PLGD may be associated with microalbuminuria; its prevalence in the wider PLGD population is unknown. Data will be collected on subjects who receive nonspecific and/or specific treatments if available; they will be analyzed for therapeutic response, disease control, and recurrence or emergence on therapy.

Further details of the methods, procedures, and planned analyses are reported in the Online Supplementary Material.

\section{Conclusions}

There is currently no central repository to collect and analyze data from people with PLGD; clinicians rely on case reports and individual experience to diagnose and treat patients, resulting in treatment variability and less than optimal outcomes. The HISTORY study may play a substantial role in addressing these deficits by collecting clinical, genetic, and laboratory data which will be analyzed to identify potential markers that can assist in disease course prediction and understanding heterogeneity in phenotypic expression. This study may also enable the development of standardized assays, the identification of at-risk groups, and guidelines for non-specific and specific therapies.

To date, no other international team of dedicated experts has been assembled to investigate the natural history of PLGD and fill knowledge gaps; HISTORY provides the first comprehensive effort to address this ultrarare disease. It is not possible for one center or country to singlehandedly complete this study; all interested parties are therefore strongly encouraged to view the study website (www.plgdeficiency.com), participate if they know of or care for anyone with PLGD or contact the authors directly to discuss potential involvement in this registry. Full enrollment and patient diversity will benefit all those with this disorder.

\section{Acknowledgments}

The authors would like to thank Ian S. Mitchell, Ph.D. and Sonia Nast, Ph.D. of GLOVAL LLC, Broomfield, CO, USA for their assistance in writing and editing this manuscript.

\section{References}

1. Forrest CB, Bartek RJ, Rubinstein Y, Groft $\mathrm{SC}$. The case for a global rare-diseases registry. Lancet. 2011;377(9771):1057-1059.

2. Jansen-van der Weide MC, Gaasterland $\mathrm{CMW}$, Roes KCB, et al. Rare disease registries: potential applications towards impact on development of new drug treatments. Orphanet J Rare Dis. 2018;13(1):154.

3. Schuster V, Hugle B, Tefs K. Plasminogen deficiency. J Thromb Haemost. 2007;5(12): 2315-2322.

4. Tait RC, Walker ID, Conkie JA, et al.
Plasminogen levels in healthy volunteers-influence of age, sex, smoking and oral contraceptives. Thromb Haemost. 1992;68(5): 506-510.

5. Tait RC, Walker ID, Conkie JA, Islam SI, $\mathrm{McC}$ all F. Isolated familial plasminogen deficiency may not be a risk factor for thrombosis. Thromb Haemost. 2018;76(6):10041008.

6. Tefs K, Tait CR, Walker ID, Pietzsch N, Ziegler M, Schuster V. A K19E missense mutation in the plasminogen gene is a common cause of familial hypoplasminogenaemia. Blood Coagul Fibrinolysis.
2003;14(4):411-416

7. Dykes D, Polesky H. Incidence of the PLG* $\mathrm{QO}$ allele in human populations. In: Mayr WR, ed. Advances in Forensic Haemogenetics: Springer-Verlag Berlin Heidelberg, 1988:261-264.

8. Okamoto A, Sakata T, Mannami T, et al. Population-based distribution of plasminogen activity and estimated prevalence and relevance to thrombotic diseases of plasminogen deficiency in the Japanese: the Suita study. J Thromb Haemost. 2003;1 (11):2397-2403.

9. Weidinger S, Patutschnick W, 
Schwarzfischer F. Further evidence of a silent plasminogen (PLG) allele in two paternity cases. Z Rechtsmed. 1988;101(2):99104

10. Mehta R, Shapiro AD. Plasminogen deficiency. Haemophilia. 2008;14(6):1261-1268.

11. Schuster V, Seregard S. Ligneous conjunctivitis. Surv Ophthalmol. 2003;48(4):369-388.

12. Klammt J, Kobelt L, Aktas D, et al. Identification of three novel plasminogen (PLG) gene mutations in a series of 23 patients with low PLG activity. Thromb Haemost. 2011;105(3):454-460.

13. Tefs K, Gueorguieva M, Klammt J, et al. Molecular and clinical spectrum of type I plasminogen deficiency: a series of 50 patients. Blood. 2006;108(9):3021-3026.

14. Shapiro AD, Nakar C, Parker JM, et al. Plasminogen replacement therapy for the treatment of children and adults with congenital plasminogen deficiency. Blood. 2018;131(12):1301-1310

15. Pantanowitz L, Bauer K, Tefs K, et al. Ligneous (pseudomembranous) inflammation involving the female genital tract associated with type-1 plasminogen deficiency. Int J Gynecol Pathol. 2004;23 (3):292-295.

16. Chi AC, Prichard E, Richardson MS, Rasenberger KP, Weathers DR, Neville BW. Pseudomembranous disease (ligneous inflammation) of the female genital tract, peritoneum, gingiva, and paranasal sinuses associated with plasminogen deficiency. Ann Diagn Pathol. 2009:13(2):132-139.

17. Cohen J, Cohen S, Cymberknoh MC, Gross M, Hirshoren N, Shoseyov D. Laryngeal obstruction in congenital plasminogen deficiency. Pediatr Pulmonol. 2012;47(9):923925.

18. Ciftci E, Ince E, Akar N, Dogru U, Tefs K, Schuster V. Ligneous conjunctivitis, hydrocephalus, hydrocele, and pulmonary involvement in a child with homozygous type I plasminogen deficiency. Eur J Pediatr. 2003;162(7-8):462-465.

19. Weinzierl MR, Collmann H, Korinth MC, Gilsbach JM, Rohde V. Management of hydrocephalus in children with plasminogen deficiency. Eur J Pediatr Surg. 2007;17(2):124-128.

20. Schott D, Dempfle CE, Beck P, et al. Therapy with a purified plasminogen concentrate in an infant with ligneous conjunctivitis and homozygous plasminogen deficiency. N Engl J Med. 1998;339(23):1679-1686.

21. Martin-Fernandez L, Marco P, Corrales I, et al. The unravelling of the genetic architecture of plasminogen deficiency and its relation to thrombotic disease. Sci Rep. 2016;6:39255.

22. Hidayat AA, Riddle PJ. Ligneous conjunctivitis. A clinicopathologic study of 17 cases. Ophthalmology. 1987;94(8):949-959.

23. De Cock R, Ficker LA, Dart JG, Garner A, Wright P. Topical heparin in the treatment of ligneous conjunctivitis. Ophthalmology. 1995;102(11):1654-1659

24. Holland EJ, Chan CC, Kuwabara T, Palestine AG, Rowsey JJ, Nussenblatt RB.
Immunohistologic findings and results of treatment with cyclosporine in ligneous conjunctivitis. Am J Ophthalmol. 1989;107(2):160-166

25. Shimabukuro $M$, Iwasaki $N$, Nagae $Y$, et al. Ligneous conjunctivitis: a case report. Jap J Ophthalmol. 2001;45(4):375-377.

26. Firat T. Ligneous conjunctivitis. Am J Ophthalmol. 1974;78(4):679-688.

27. Sartori MT, Saggiorato G, Pellati D, et al. Contraceptive pills induce an improvement in congenital hypoplasminogenemia in two unrelated patients with ligneous conjunctivitis. Thromb Haemost. 2003;89(01):86-91

28. Fine G, Bauer K, Al-Mohaya M, Woo SB. Successful treatment of ligneous gingivitis with warfarin. Oral Surg Oral Med Oral Pathol Oral Radiol Endod. 2009;107(1):77-

29. Barabino S, Rolando M. Amniotic membrane transplantation in a case of ligneous conjunctivitis. Am J Ophthalmol. 2004;137 (4):752-753

30. Tabbara KF. Prevention of ligneous conjunctivitis by topical and subconjunctival fresh frozen plasma. Am J Ophthalmol. 2004;138 (2):299-300

31. Kizilocak H, Ozdemir N, Dikme G, et al. Treatment of plasminogen deficiency patients with fresh frozen plasma. Pediat Blood Cancer. 2018:65(2):e26779.

32. Watts P, Suresh P, Mezer E, et al. Effective treatment of ligneous conjunctivitis with opical plasminogen. Am J Ophthalmol. 2002;133(4):451-455

33. Pons V, Olivera P, Garcia-Consuegra R, et al. Beyond hemostasis: the challenge of treating plasminogen deficiency. A report of three cases. J Thromb Thrombolysis. 2016:41 (3):544-547.

34. Nakar CT, Caputo R, Price FW, et al. Safety \& efficacy of human plasma derived plasminogen ophthalmic drops for treatment of ligneous conjunctivitis: report of phase $2 / 3$ clinical trial. Blood. 2015;126(23):2288.

35. O'Mahony B, Noone D, Giangrande PL, Prihodova L. Haemophilia care in Europe survey of 35 countries. Haemophilia. 2013:19(4):e239-247.

36. Keipert C, Hesse J, Haschberger B, et al. The growing number of hemophilia registries: quantity vs, quality. Clin Pharmacol Ther. 2015;97(5):492-501

37. Osooli M, Berntorp E. Inhibitors in haemophilia: what have we learned from registries? A systematic review. J Intern Med. 2015;277(1):1-15.

38. European Medicines Agency. Report on Haemophilia Registries; Workshop 8 June 2018; Patient Registries Initiative. [cited September 23, 2019]; Available from: https://www.ema.europa.eu/en/docu ments/report/report-haemophilia-registriesworkshop en.pdf

39. Gupta S, Acharya S, Roberson C, Lail A, Soucie JM, Shapiro A. Potential of the Community Counts registry to characterize rare bleeding disorders. Haemophilia. 2019;25(6):1045-1050.
40. Konkle BA, Johnsen JM, Wheeler M, et al. Genotypes, phenotypes and whole genome sequence: approaches from the My Life Our Future haemophilia project. Haemophilia. 2018;24 Suppl 6:87-94.

41. Centers for Disease Control and Prevention Community Counts. [cited September 24, 2019]; Available from: https://www.cdc.gov/ncbddd/hemophilia/c ommunitycounts/data-reports/2018 9/table-1-patient-characteristics-by-calendar.html

42. Peyvandi F, Palla R, Menegatti M, et al Coagulation factor activity and clinical bleeding severity in rare bleeding disorders: results from the European Network of Rare Bleeding Disorders. J Thromb Haemost. 2012;10(4):615-621.

43. Peyvandi F, Di Michele D, Bolton-Maggs $\mathrm{PH}$, et al. Classification of rare bleeding disorders (RBDs) based on the association between coagulant factor activity and clinical bleeding severity. J Thromb Haemost. 2012;10(9):1938-1943.

44. European Association for Haemophilia and Allied Disorders. Prospective Rare Bleeding Disorders Database (PRO-RBDD). [cited September 23, 2019]; Available from: http://eahad.org/prospective-rare-bleedingdisorders-database-pro-rbdd/

45. Menegatti M, Palla R, Boscarino $M$, et al Minimal factor XIII activity level to preven major spontaneous bleeds. I Thromb Haemost. 2017;15(9):1728-1736.

46. Iorio A, Keepanasseril A, Foster G, et al Development of a Web-Accessible Population Pharmacokinetic ServiceHemophilia (WAPPS-Hemo): study protocol. JMIR Res Protoc. 2016;5(4):e239.

47. Hemker HC, Giesen P, Al Dieri R, et al. Calibrated automated thrombin generation measurement in clotting plasma. Pathophysiol Haemost Thromb. 2003;33 (1):4-15

48. Chitlur M, Sorensen B, Rivard GE, et al. Standardization of thromboelastography: a report from the TEG-ROTEM working group. Haemophilia. 2011;17(3):532-537.

49. van Geffen M, Loof A, Lap P, et al. A novel hemostasis assay for the simultaneous measurement of coagulation and fibrinolysis. Hematology. 2011;16(6):327-336.

50. Colucci M, Binetti BM, Tripodi A Chantarangkul V, Semeraro N Hyperprothrombinemia associated with prothrombin G20210A mutation inhibits plasma fibrinolysis through a TAFI-mediated mechanism. Blood, 2004:103(6):21572161

51. Ma Q, Ozel AB, Ramdas S, et al. Genetic variants in PLG, LPA, and SIGLEC 14 as well as smoking contribute to plasma plasminogen levels. Blood. 2014;124(20):3155-3164.

52. Li JF, Lin Y, Yang YH, et al. Fibrinogen Aalpha Thr312Ala polymorphism specifically contributes to chronic thromboembolic pulmonary hypertension by increasing fibrin resistance. PLoS One. 2013;8 (7):e69635 